\title{
MAPK pathway contributes to density- and hypoxia-induced expression of the tumor-associated carbonic anhydrase IX
}

\author{
Juraj Kopacek ${ }^{\mathrm{a}, \mathrm{b}}$, Monika Barathova ${ }^{\mathrm{a}}$, Franck Dequiedt ${ }^{\mathrm{c}}$, Janka Sepelakova ${ }^{\mathrm{a}}$, Richard Kettmann ${ }^{\mathrm{c}}$, \\ Jaromir Pastorek $^{\mathrm{a}}$, Silvia Pastorekova, ${ }^{\mathrm{a}, *}$ \\ ${ }^{a}$ Centre of Molecular Medicine, Institute of Virology, Slovak Academy of Sciences, Dubravska cesta 9, 84505 Bratislava, Slovakia \\ ${ }^{\mathrm{b}}$ Department of Microbiology and Virology, Faculty of Natural Sciences, Comenius University, Bratislava 842 15, Slovakia \\ ${ }^{\mathrm{c}}$ Faculty of Agronomy, Department of Applied Biochemistry and Biology, Avenue Marechal Juin 13, 5030 Gembloux, Belgium
}

Received 6 December 2004; received in revised form 21 February 2005; accepted 8 March 2005

Available online 23 March 2005

\begin{abstract}
Transcription of the CA9 gene coding for a tumor-associated carbonic anhydrase IX (CA IX) isoform is regulated by hypoxia via the hypoxia-inducible factor 1 (HIF-1) and by high cell density via the phosphatidylinositol-3-kinase (PI3K) pathway. We examined the role of the mitogen-activated protein kinase (MAPK) pathway in the control of CA9 gene expression. Inhibition of MAPK signaling by U0126 in HeLa cells led to reduced activity of the PR1-HRE-luc CA9 promoter construct and decreased CA IX protein levels in dense culture as well as in hypoxia. Similar reduction was obtained by expression of a dominant-negative ERK1 mutant and was also observed in U0126-treated HIF-1 $\alpha$-deficient Ka13 cells. Simultaneous treatment with the MAPK and PI3K inhibitors U0126 and LY 294002 had stronger effect than individual inhibition of these pathways. Taken together, our results suggest that besides the PI3K pathway, the MAPK cascade is involved in the regulation of $C A 9$ gene expression under both hypoxia and high cell density.
\end{abstract}

(C) 2005 Elsevier B.V. All rights reserved.

Keywords: Carbonic anhydrase IX; Hypoxia; Cell density; MAPK pathway; Promoter activity; Transcriptional regulation

\section{Introduction}

Carbonic anhydrases are widely distributed enzymes, which play important roles in diverse physiological and pathological processes. Via catalysis of a reversible conversion of carbon dioxide to bicarbonate and proton, they participate in gas exchange, ion transport and acidbase balance across the cell membrane and in different intracellular compartments. Eleven out of twelve enzymatically active mammalian isoforms are predominantly associated with differentiated cells and fulfill specialized

Abbreviations: CA IX, carbonic anhydrase protein; CA9, carbonic anhydrase gene; HIF, hypoxia inducible factor; PI3K, phosphatidylinositol3-kinase; MAPK, mitogen-activated kinase

* Corresponding author. Fax: +42125477 4284

E-mail address: virusipa@savba.sk (S. Pastorekova). functions in various tissues and organs [1]. In contrast, the carbonic anhydrase IX (CA IX) isoenzyme is mainly present in solid tumors and its expression in normal tissues is limited to the epithelia of the gastrointestinal tract [2]. Moreover, expression of CA IX is linked with poor prognosis in several types of carcinomas [3], and the protein appears to be functionally implicated in tumorigenesis as a part of the regulatory mechanisms that control $\mathrm{pH}$ and cell adhesion [4,5].

Strong association of CA IX with a broad range of tumors is principally related to its transcriptional regulation by hypoxia and high cell density, which appear to activate the $C A 9$ promoter through two different, but interconnected pathways [6-8]. The promoter of the CA9 gene contains five regions protected in DNase I footprinting (PR1-PR5, numbered from the transcription start) [9]. PR1 and PR2 bind SP1/3 and AP1 transcription factors and are critical for the basic activation of $C A 9$ transcription $[9,10]$. 
The CA9 promoter sequence $(-3 /-10)$ between the transcription start and PR1 contains a HRE element recognized by a hypoxia inducible factor 1 (HIF-1), which governs transcriptional responses to hypoxia [6]. HIF-1 is composed of two subunits: a constitutively expressed HIF-1 $\beta$ and a rate-limiting HIF- $1 \alpha$, which is regulated by the availability of oxygen. Under hypoxia, HIF-1 $\alpha$ skips modification of its conserved proline and asparagine residues by oxygen-sensitive hydroxylases, thus avoiding degradation mediated by pVHL and inactivation mediated by FIH-1 [11-13]. This leads to HIF- $1 \alpha$ accumulation, dimerization with HIF- $1 \beta$, binding to HRE sites in the target genes, interaction with the cofactors and stimulation of the HIF-1 trans-activation capacity. In addition, HIF- $1 \alpha$ can be up-regulated under normoxic conditions by different extracellular signals and oncogenic changes transmitted via the PI3K and MAPK pathways $[14,15]$. Whereas PI3K activation results in an increased level of HIF- $1 \alpha$ protein, MAPK activation improves its trans-activation properties [16,17].

HIF-1 strongly induces transcription of the CA9 gene in hypoxia, but for full induction requires a contribution of the SP1/3 transcription factor binding to PR1 $[6,18]$. Upregulation of CA9 transcription in increased cell density involves a mild pericellular hypoxia, depends upon cooperation of SP1 with HIF-1 at subhypoxic level and operates via the PI3K pathway [8]. Hypoxia and cell density act in an additive fashion so that the highest expression of $C A 9$ is achieved under conditions of low oxygen at high density [8].

In this work, we studied a role of the MAPK pathway in the transcriptional activation of the CA9 gene. The rationale was based on numerous data demonstrating an importance of the MAPK pathway in both hypoxiadependent and -independent activation of HIF-1 as well as in the regulation of its transcriptional targets, such as VEGF [17,19-21]. In accord with those studies, we found that inhibition of the MAPK pathway by a specific inhibitor down-regulated the $C A 9$ promoter activity and CA IX protein expression in both hypoxia and high cell density. Transcriptional activity of the $C A 9$ promoter was also reduced by expression of a dominant-negative mutant of the ERK1 component of the MAPK pathway. Finally, we showed that a simultaneous inhibition of PI3K and MAPK signaling down-regulated the $C A 9$ promoter activity and protein level more strongly than their separate inhibition suggesting a concurrent involvement of these pathways in the control of the CA9 expression.

\section{Materials and methods}

\subsection{Cell culture and hypoxic treatment}

HeLa cells derived from human cervical carcinoma and HEK293 human embryonic kidney cells were cultured in
DMEM supplemented with 10\% FCS (BioWhittaker, Verviers, Belgium) under humidified air containing 5\% $\mathrm{CO}_{2}$ at $37{ }^{\circ} \mathrm{C}$. Ka13 derivative of CHO-K1 Chinese hamster cells (kindly provided by Dr. Patrick Maxwell, Imperial College of Science, Technology and Medicine, London, UK) [22] were cultured in Ham's F12 medium with $10 \%$ FCS. The cells were exposed to hypoxia $(1 \%$ $\mathrm{O}_{2}$ ) in an anaerobic workstation (Ruskin Technologies, Bridgend, UK) in $5 \% \mathrm{CO}_{2}, 10 \% \mathrm{H}_{2}$ and $84 \% \mathrm{~N}_{2}$ at 37 ${ }^{\circ} \mathrm{C}$. Hypoxia was also induced chemically either with 200 $\mu \mathrm{M}$ deferoxamine mesylate (DFO, Sigma, St. Louis, MO), an iron chelator commonly used in the study of hypoxiainduced responses, or with $0.75 \mathrm{mM}$ 2-oxoglutaratedependent dioxygenase inhibitor dimethyloxalylglycine (DMOG, Frontier Scientific, Logan UT).

\subsection{Inhibitors, antibodies and plasmids}

The MAPKK inhibitor U0126 (Sigma), the PI3K inhibitor LY294002 (Calbiochem, Cambridge, MA) and the SP1 inhibitor mithramycin A (MMA, Sigma) were dissolved in dimethyl sulfoxide (DMSO) at $10 \mathrm{mM}$, and stored in aliquots at $-20{ }^{\circ} \mathrm{C}$. Prior to use, the inhibitors were diluted in culture medium to working concentrations, i.e. $20 \mu \mathrm{M} \mathrm{U} 0126,10 \mu \mathrm{M}$ LY294002 and $100 \mathrm{nM}$ MMA. The final concentration of DMSO was less than $0.2 \%$ including controls. Cultures were pre-incubated with the inhibitors $1 \mathrm{~h}$ before the induction of hypoxia or addition of DFO. Cytotoxic drug effects were monitored by the colorimetric Cell Titer Blue method (Promega, Madison, WI).

M75 mouse monoclonal antibody specific for the Nterminal PG region of the CA IX protein was described previously $[23,24]$. Secondary anti-mouse antibodies conjugated with horse-radish peroxidase were purchased from Sevapharma (Prague, Czech Republic).

The PR1-HRE-luc promoter construct was generated by an insertion of a $-50 /+37 C A 9$ genomic region amplified by PCR upstream of the firefly luciferase gene in pGL3-Basic luciferase reporter vector (Promega). pRLTK renilla vector (Promega) served for the control of the transfection efficiency. HIF- $1 \alpha$ cDNA in pcDNA1/Neo/ HIF-1a expression plasmid [22] was kindly provided by Dr. Patrick Maxwell. Dominant-negative mutants of ERK1 (pcDNA-ERK1) and ERK2 (pcDNA-ERK2) mutated in their ATP binding sites were kindly provided by Dr. M.H. Cobb (Southwestern Medical Center, Dallas) [20].

\subsection{Transient transfection and luciferase assay}

The cells were plated into $30 \mathrm{~mm}$ Petri dishes to reach approximately $60 \%$ monolayer density on the next day. Transfection was performed with the $2 \mu \mathrm{g}$ of PR1-HREluc and $100 \mathrm{ng}$ of pRL-TK plasmids DNAs using a GenePorterII reagent (Gene Therapy Systems, San Diego, CA) according to the manufacturer's recommendation. One day later, the transfected cells were trypsinized and 
plated in triplicates into 24 -well plates at different densities so that the dense culture contained eight times more cells than the sparse one. The cells were allowed to attach for $20 \mathrm{~h}$, then they were pre-treated with inhibitors for $1 \mathrm{~h}$ and transferred to hypoxia (or treated with DFO) for additional $24 \mathrm{~h}$. Reporter gene expression was assessed $48 \mathrm{~h}$ after the transfection using the DualLuciferase Reporter Assay System (Promega) and the luciferase activity was normalized against the renilla expression.

\subsection{Immunoblotting}

HeLa cells were plated in dense $\left(80,000\right.$ cells $\left./ \mathrm{cm}^{2}\right)$ and sparse $\left(10,000\right.$ cells $\left./ \mathrm{cm}^{2}\right)$ cultures and incubated for $24 \mathrm{~h}$. Then the cells were pre-treated with inhibitors for $1 \mathrm{~h}$ and transferred to hypoxia for $24 \mathrm{~h}$. Parallel control dishes were pre-treated and maintained in normoxia for the same time period.

For the detection of CA IX, the cells were extracted with cold RIPA buffer for 15 min at $4{ }^{\circ} \mathrm{C}$. The extracts were then centrifuged (15 $\mathrm{min}$ at $13,000 \mathrm{rpm})$ and total protein concentrations were determined by BCA assay (Pierce, Rockford, IL). Samples of $30 \mu \mathrm{g}$ total proteins were separated by the electrophoresis using 10\% SDS-PAGE and blotted onto the PVDF membrane. Before immunodetection, the membrane was treated by the blocking buffer containing 5\% non-fat milk in PBS with $0.2 \%$ Nonidet P-40 for $1 \mathrm{~h}$ and incubated for $1 \mathrm{~h}$ with M75 MAb diluted 1:2 in the blocking buffer. Then the membrane was washed, incubated for $1 \mathrm{~h}$ with the anti-mouse secondary antibody, washed again and developed with the ECL detection system. Intensity of CA IX-specific bands was analyzed by the Scion Image Beta 4.02 software (Scion Corporation, Frederick, MD) and relative CA IX expression was expressed in percent.

\section{Results}

3.1. Inhibition of MAPKK reduces CA9 promoter activity and $C A I X$ protein expression in both hypoxia and high density

Previous studies have determined PR1-HRE as a crucial cell density- and hypoxia-inducible module within the $C A 9$ promoter $[8,18]$. Therefore, we have cloned a $-50 /+37$ CA9 genomic region, containing this module in the natural context of the transcription start site, upstream of the reporter luciferase gene. The PR1-HRE-luc promoter construct was then co-transfected with the renilla-coding control plasmid to HeLa cells that express CA IX protein in response to hypoxia and high cell density. In accord with earlier observations, the highest CA9 promoter activity was obtained in a dense culture exposed to hypoxia-mimicking agent DFO. Treatment of the cells with a MAPKK inhibitor U0126 resulted in about a fourfold decrease of the $C A 9$ promoter activity irrespective of the conditions used for cell incubation (Fig. 1A). CA9 promoter induction and its U0126 inhibition were comparable in physiological hypoxia (not shown). In addition, endogenous CA IX protein levels produced in HeLa cells upon MAPKK inhibition under hypoxia and/or high density corresponded with the promoter activities (Fig. 1B). Similar results were obtained when PR1-HRE-luc construct was transfected to HEK293 cells that do not express endogenous CA IX protein, but contain the transcriptional machinery needed for the activation of the CA9 promoter by hypoxia as well as by high cell density. The increase of the $C A 9$ promoter activity observed in a
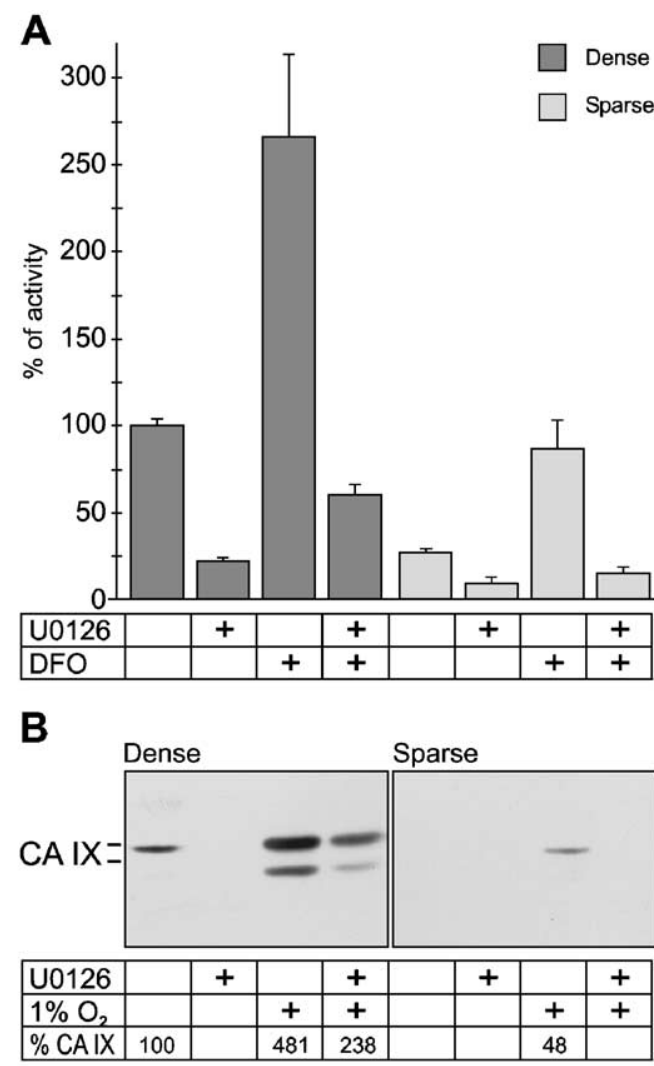

Fig. 1. Effect of MAPK pathway inhibition by U0126 on $C A 9$ promoter activity and CA IX protein level. (A) Transcriptional activity of PR1HRE-luc portion of the $C A 9$ promoter in HeLa cells grown in sparse and dense cultures. The cells were co-transfected with PR1-HRE-luc CA9 promoter construct and renilla plasmid, re-plated at different densities, pre-treated with U0126 MAPK inhibitor and subjected to DFO-induced hypoxia. CA9 promoter activity was measured $48 \mathrm{~h}$ after the transfection and calculated as a ratio between the luciferase and renilla-related values. Results are expressed as the percentage of activity obtained in dense normoxic cultures. Bars represent the mean values including standard deviations. (B) Expression of endogenous CA IX protein in U0126treated HeLa cells grown in high and low density and under normoxia $\left(\begin{array}{lll}20 \% & \mathrm{O}_{2}\end{array}\right)$ and atmospheric hypoxia $\left(\begin{array}{lll}1 \% & \mathrm{O}_{2}\end{array}\right)$ was analyzed by immunoblotting using CA IX-specific monoclonal antibody M75. Relative level of CA IX protein was analyzed by Scion Image and all measurable values were expressed in percent (\% CA IX). 

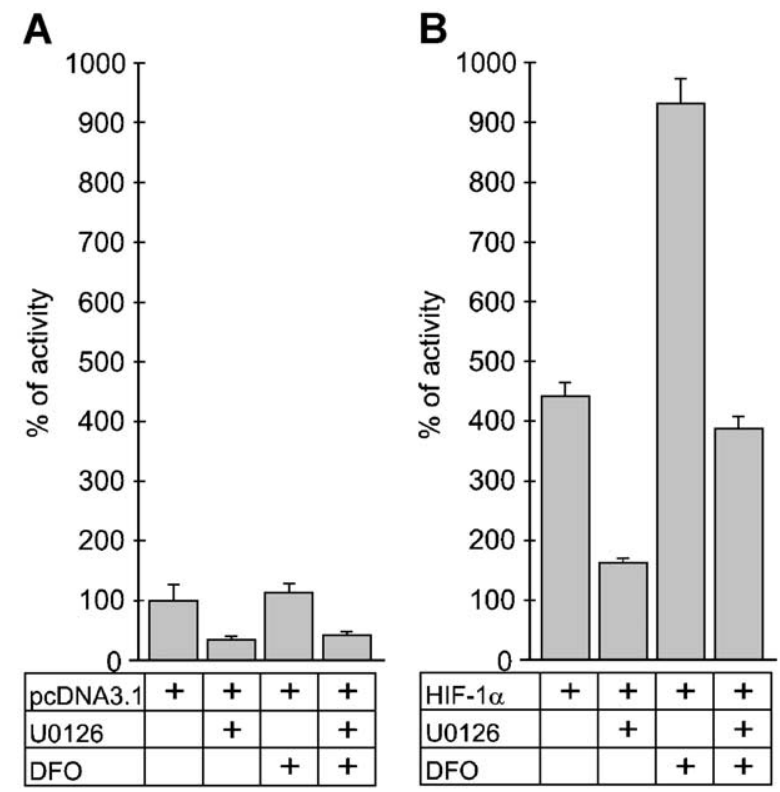

Fig. 2. Transcriptional activity of the CA9 promoter in Ka13 cells cotransfected with the empty pcDNA3.1 plasmid (A) and with the plasmid encoding HIF-1 $\alpha$ cDNA (B). Transfection with PR1-HRE-luc construct, treatment with U0126 and DFO, cell cultivation and assessment of the CA9 promoter activity was as described in Fig. 1. Results are expressed as the mean percentage of activity obtained in the normoxic cultures transfected with the empty plasmid. Bars represent the mean values including standard deviations.

dense culture exposed to hypoxia was considerably inhibited by treatment with the U0126 inhibitor of MAPKK (data not shown).

\subsection{Inhibition of MAPKK reduces CA9 promoter activity in

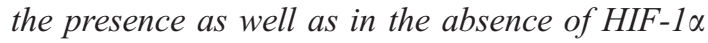

Next we sought to find out whether regulation of $C A 9$ expression by the MAPK pathway depends on the presence of HIF-1 $\alpha$. For this purpose we used CHOderived Ka13 cells that do not express endogenous HIF$1 \alpha$ protein [22]. These HIF-1 $\alpha$-deficient cells were previously shown to be unable to normally activate the CA9 promoter in response to cell density [8]. Therefore, we plated the Ka13 cells at intermediate density, transfected them with PR1-HRE-luc promoter construct together with the renilla control plasmid and pcDNA3.1 plasmid, pre-treated with U0126 MAPKK inhibitor and exposed to a DFO-induced hypoxia. Parallel dishes were maintained in absence of DFO. Expectedly, the CA9 promoter activity was not increased in hypoxia apparently due to the absence of the HIF- $1 \alpha$ protein. In spite of this, inhibition of MAPKK by U0126 diminished the promoter activity to less than a half in both conditions (Fig. 2A). Co-transfection of the PR1-HRE-luc construct with a cDNA encoding the wild-type HIF-1 $\alpha$ led to a remarkable elevation of the $C A 9$ promoter activity, which was further increased upon DFO-induced hypoxia, possibly as a result of the stabilization of the ectopically expressed HIF- $1 \alpha$ (Fig. 2B). In correspondence with the results of the previous experiments, MAPKK inhibition reduced the promoter activity to approximately one third of a HIF-1 $\alpha$ induced value. These data indicate that MAPK pathway can affect the CA9 expression both via a HIF-1-mediated
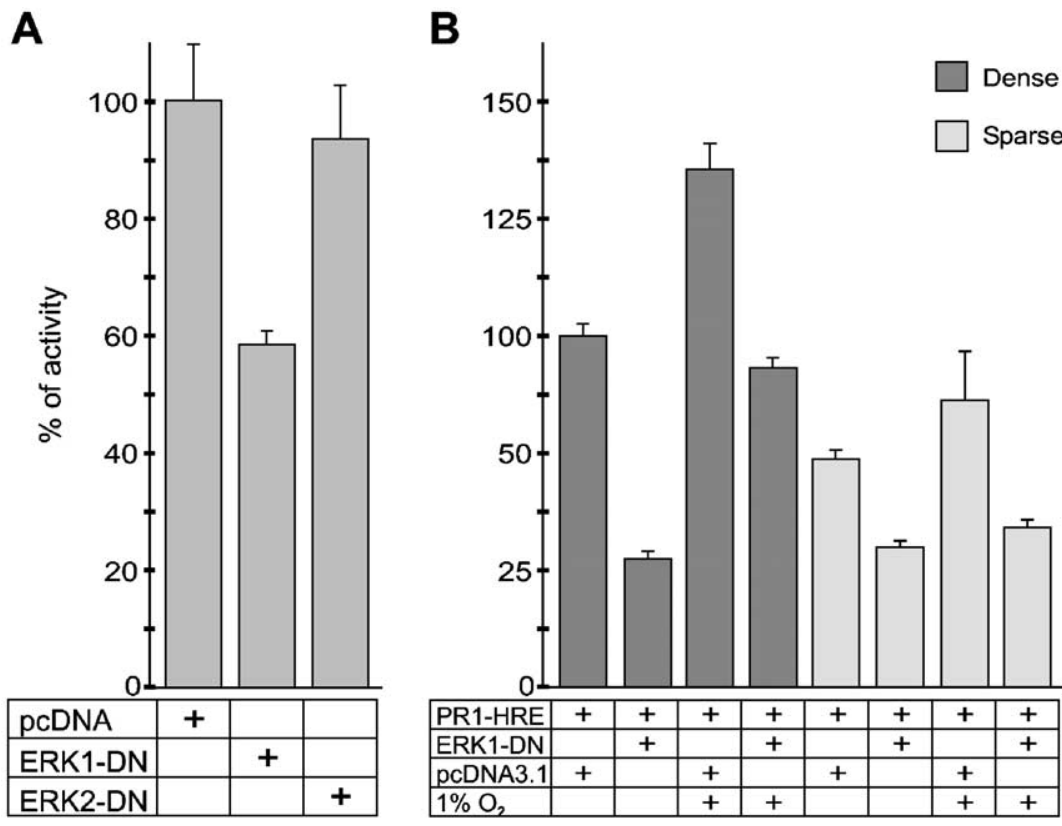

Fig. 3. Influence of dominant-negative mutant of MAPK/ERK1 on the CA9 promoter activity in HEK293 cells. (A) The cells were co-transfected with the PR1HRE-luc promoter construct and cDNA encoding the dominant-negative mutants of MAP kinases ERK1 (ERK1-DN) and ERK2 (ERK2-DN) and maintained in normoxia under high density. (B) The cells were co-transfected with PR1-HRE-luc and ERK1-DN plasmids and grown in sparse or dense culture under normoxia or hypoxia $\left(1 \% \mathrm{O}_{2}\right)$. Transcriptional activity of the $C A 9$ promoter was determined as described in Fig. 1. Results are expressed as the mean percentage of activity obtained in the cells co-transfected with an empty pcDNA3.1 plasmid that served as a control. 
transcriptional activation and via a HIF-1 $\alpha$-independent mechanism.

\subsection{CA9 promoter activity decreases upon expression of a dominant negative mutant of ERK1}

MAPKK signaling is transmitted essentially via two downstream mediators, namely the serine/threonine kinases MAPK/ERK1 and MAPK/ERK2 [25]. To learn, which of the two ERKs is involved in the control of $C A 9$ expression, we co-transfected the PR1-HRE-luc plasmid with the plasmids encoding the dominant-negative (DN) kinase-dead mutants of either ERK1 or ERK2 into dense HEK293 cells. These cells were chosen for this experiment due to their capacity to allow for a very high efficiency in transient co-transfection and for their full competence to drive transcription from the $C A 9$ promoter as mentioned above. On the other hand, HeLa cells have a high basal level of MAPK activity even in the absence of serum [26] and transient co-transfection with DN mutants is not sufficient to get consistent results. Luciferase activities obtained in the transfected HEK293 cells and normalized against the renilla control revealed that co-expression of ERK1-DN reduced the CA9 promoter activity by about $40 \%$, whereas co-expression of ERK2-DN had no significant effect (Fig. 3A). Based on this finding, we performed a co-transfection of PR1HRE-luc with ERK1-DN to the cells grown in sparse and dense conditions under normoxia and hypoxia. As expected, expression of a kinase-dead mutant of ERK1 negatively affected the $C A 9$ promoter activity in all examined conditions (Fig. 3B). Hypoxic induction of the $C A 9$ promoter was not as dramatic as seen before in HeLa cells, possibly due to a lower level of HIF-1 $\alpha$ protein in HEK293 cells [17]. Nevertheless, these findings clearly suggested that ERK1 participates in the MAPK pathway-related molecular mechanisms regulating the expression of $C A 9$.

\subsection{Simultaneous inhibition of MAPK and PI3K pathways has an additive negative effect on CA9 promoter activity and $C A$ IX protein expression}

Comparison of the normoxic and hypoxic activities of the $C A 9$ promoter in all HeLa, HEK293 and Ka13 cell lines has shown that the U0126-treatment did not completely abolish the induction of $C A 9$ expression. This fact indicated that a part of the regulatory mechanisms, which transmit molecular signals generated by hypoxia and/or high cell density, remained functional. Previous studies provide evidence for the involvement of the PI3K pathway in the density-induced CA IX expression [8]. We therefore anticipated that this $\mathrm{PI} 3 \mathrm{~K}$ pathway could be responsible for the $C A 9$ promoter activity remaining after inhibition of the MAPK signaling. To examine this assumption, HeLa cells incubated in normoxia and physiological hypoxia were treated with inhibitors of MAPK (U0126) and PI3K pathways (LY294002). The inhibitors were tested to determine the concentration that would give the maximum combined inhibitory effect without compromising cell survival (data not shown). Each inhibitor alone was able to reduce the $C A 9$ promoter activity measured in dense hypoxic HeLa cells transfected with PR1-HRE-luc construct to about one third of its control value and their simultaneous effect was still stronger (Fig. 4A). The effects of inhibitors were less pronounced in the normoxic and sparse cells, but showed similar tendency. Immunoblotting analysis of
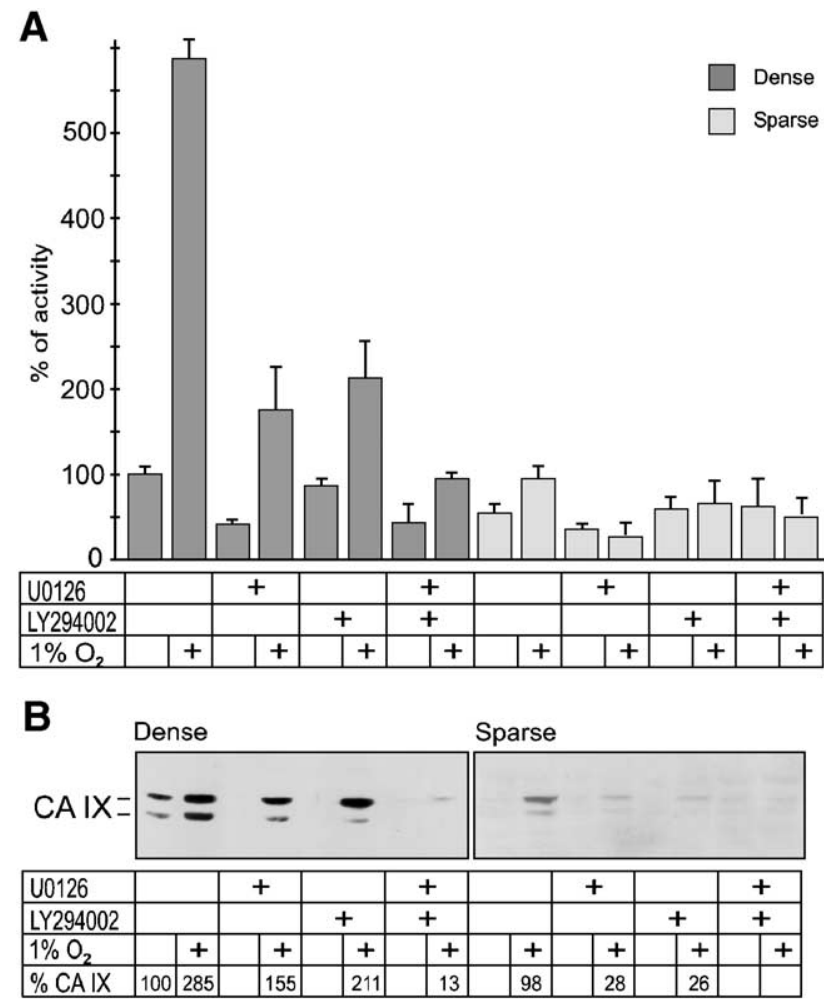

C

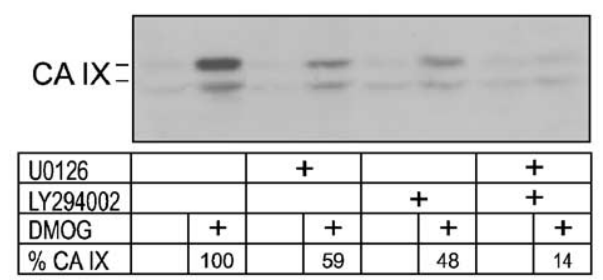

Fig. 4. Expression of $C A 9$ gene in HeLa cells treated with inhibitors of both MAPK and PI3K pathways. (A) Effect of inhibitors on the CA9 promoter activity was evaluated in the cells transfected with the PR1HRE-luc promoter construct, re-plated at high or low density and maintained for $24 \mathrm{~h}$ in normoxia or hypoxia $\left(1 \% \mathrm{O}_{2}\right)$. Results are expressed as the mean percentage of activity measured in the non-treated cells and include standard deviations. (B) Sparse and dense HeLa cells were incubated for $48 \mathrm{~h}$ under normoxia and hypoxia in the presence of U0126 and LY294002 inhibitors. Cell extracts were analyzed by immunoblotting with M75 MAb to visualize the resulting level of CA IX protein. (C) The same experiment was performed in sparse HeLa cells using DMOG dioxygenase inhibitor that stabilizes HIF-1 $\alpha$. Relative level of CA IX protein was analyzed by Scion Image and all measurable values were expressed in percent (\% CA IX). 
endogenous CA IX protein expression in HeLa cells treated with the inhibitors confirmed that CA IX protein level was considerably diminished by the LY94002 inhibitor alone and addition of the U0126 inhibitor caused its further decrease (Fig. 4B). This effect could be observed under both high and low cell density. The inhibitors similarly reduced CA IX protein expression induced in HeLa cells by DMOG, a hydroxylase inhibitor that increases stability and activity of HIF-1 $\alpha$ (Fig. 4C). Altogether, these results allowed us conclude that both PI3K and MAPK pathways act in parallel to activate the $C A 9$ gene both in hypoxia and in high cell density.

\subsection{Inhibition of SP1 activity further reduces CA9 gene expression induced by hypoxia and/or high cell density}

Our findings presented above suggest that inhibition of MAPKK interfered with a principal activating mechanism that functions under low oxygen supply as well as in the normoxic cells grown in a dense culture. The triggered signal transduction pathways seem to be integrated by a
PR1-binding SP1 transcription factor, which was shown to be required for the cooperative interaction with HREbinding HIF-1 $\alpha$ under both conditions [18]. Therefore, we treated PR1-HRE-luc-transfected HeLa cells with SP1 inhibitor MMA and with MAPKK inhibitor U0126. This experiment included also PI3K inhibitor LY294002 combined with MMA. Treatment of the transfected cells with $\mathrm{MMA}+\mathrm{U} 0126$ and MMA + LY29002, respectively, resulted in stronger inhibition of CA9 promoter activity when compared to MMA alone. Simultaneous addition of all inhibitors showed an augmented effect, which was especially marked in dense hypoxic culture (Fig. 5A). Furthermore, each MMA and U0126 separately reduced the CA IX protein level, but the combination of inhibitors almost completely prevented the hypoxic induction of CA IX protein expression (Fig. 5B). Inhibition of HeLa cells grown in a dense culture gave very similar results. Our data confirm that SP1 mediates both hypoxia and density induced trans-activation signals as it was already proposed by Kaluz et al. [18]. They also indicate that CA9 gene expression accepts signals transmitted by PI3K and MAPK
A

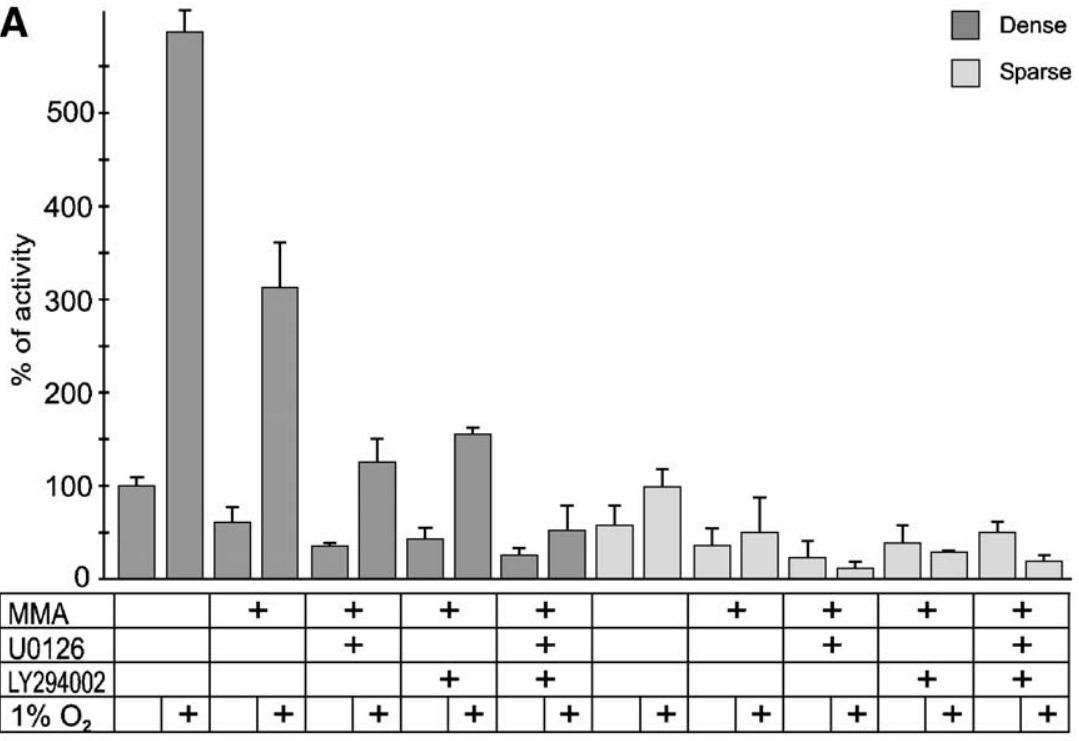

B

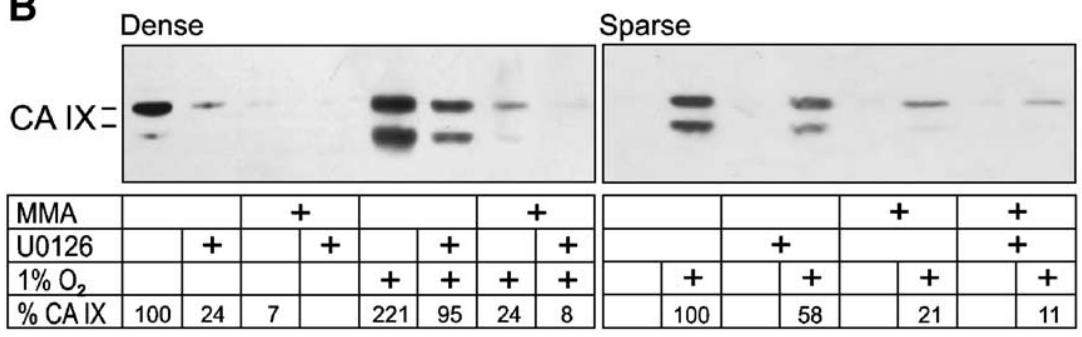

Fig. 5. Excessive negative effect of SP1 inhibition on the CA9 promoter activity and CA IX protein expression in MAPK-and/or PI3K-inhibited cells. (A) Effect of inhibitors on the CA9 promoter activity was evaluated in HeLa cells transfected with the PR1-HRE-luc promoter construct, re-plated at high/low density and exposed to normoxia/hypoxia $\left(1 \% \mathrm{O}_{2}\right)$. Results are expressed as the mean percentage of the activity measured in the non-treated cells and include standard deviation. (A) HeLa cells were seeded at low and high density. After the pre-treatment with the inhibitors, the cells were transferred to hypoxia (1\% $\mathrm{O}_{2}$ ), and parallel dished were kept in normoxia. After $48 \mathrm{~h}$, the cells were extracted and analyzed by immunoblotting. Relative level of CA IX protein was analyzed by Scion Image and all measurable values were expressed in percent (\% CA IX). 
at least partially via SP1 and that these paths may overlap and/or complement each other in regulation of the $C A 9$ promoter.

\section{Discussion}

The MAPK pathway plays an important role in transduction of extracellular signals exerted by various mitogenic and micro-environmental factors [25]. Hypoxia, which is frequently present in tumor microenvironment, activates ERK kinases by inducing their phosphorylation and nuclear translocation $[20,27]$. ERKs then increase a trans-activation capacity of HIF-1 by transmitting the phosphorylation signal to the C-terminal domain of HIF-1 $\alpha$ subunit [20] as well as by recruitment and phosphorylation of HIF-1 coactivators $[28,29]$. Under normoxic conditions, the MAPK pathway becomes activated by various growth factors, hormones and by high cell density $[25,30]$. This activation has similar effects on HIF- $1 \alpha$ and also promotes transcription of HIF-1-regulated genes [17]. Depending on the cell type and culture conditions, hypoxia and mitogenic stimulation can work together to enhance the activation of the MAPK pathway and up-regulation of HIF-1 activity. It is not surprising that this pathway significantly influences the expression of the HIF-1 targets, such as VEGF [31]. Nevertheless, HIF- $1 \alpha$ is not the only transcription factor regulated by MAPK, and HIF-1-independent mechanisms of MAPK-regulated expression of different genes including VEGF have been described $[19,27]$.

In this study we analyzed the transcriptional regulation of the CA9 gene that is a direct target of HIF-1, which binds to the HRE element adjacent to the transcription initiation site. Hence, $C A 9$ is strongly induced by hypoxia [6]. In addition, $C A 9$ transcription can be up-regulated under normoxic conditions by a high cell density [7]. This density-induced $C A 9$ expression involves a pericellular hypoxia, depends on subhypoxic levels of HIF-1 and is mediated by PI3K signaling [8]. PI3K is a key component of another signal transduction pathway that is activated under hypoxia, up-regulates HIF-1 by increasing protein synthesis of HIF-1 $\alpha$ subunit and can also transmit extracellular signals in a HIF-1 independent manner $[16,32,33]$. The list of targets involves VEGF that is expressed in response to activation of the PI3K pathway under hypoxia as well as under normoxia [33-36]. Altogether, VEGF expression is subjected to a complex regulation by at least three major signal transduction pathways driven by HIF-1, PI3K and MAPK.

Based on the principal significance of the MAPK pathway in regulation of gene expression in diverse cellular processes and using the VEGF gene as a paradigm, we decided to investigate whether the MAPK pathway contributes to control of $C A 9$ transcription. If so, we also wanted to know whether MAPK signaling is important either for hypoxic induction of $C A 9$ expression or for its up-regulation by density, or for both. Therefore, we analyzed the activity of a crucial PR1-HRE portion of the CA9 promoter in cells grown in low and high densities as well as in those maintained in low and normal oxygen levels. The cells transfected by the PR1-HRE-luc promoter construct were re-plated to different densities, pre-treated with the MAPKK U0126 inhibitor and then subjected to hypoxia. In both HeLa and HEK293 cell lines used, we observed a remarkable reduction of the $C A 9$ promoter activity following inhibition of the MAPK pathway in all tested conditions of cell incubation. The same expression pattern was obtained with an endogenous CA IX protein expressed in the MAPK inhibitor-treated HeLa cells. These results have shown that our assumption was correct and that the MAPK pathway is actually involved in the control of CA9 gene expression both in hypoxia and high cell density.

Of course, effects of the MAPK pathway inhibition could rely on HIF-1 as it was mentioned above and as it was also shown for the PI3K pathway in the density-induced $C A 9$ expression [8]. Indeed, involvement of HIF-1 was indirectly supported by the finding of a negative regulation of the $C A 9$ promoter activity by a dominant-negative mutant of MAPK/ ERK1, but not ERK2, since only ERK1 can phosphorylate and activate HIF-1 $\alpha$ [20]. However, examination of the CA9 promoter activity in HIF- $1 \alpha$ deficient Ka13 cells and in the same cells co-transfected with the HIF-1 $\alpha$ cDNA revealed that at least a part of the response to MAPK inhibition is not dependent on HIF- $1 \alpha$. In the absence of HIF- $1 \alpha$, Ka13 cells are unable to induce $C A 9$ expression in hypoxia and a high cell density has only a weak stimulatory effect [8], but the treatment with the MAPK inhibitor can lower even the basal CA9 promoter activity to less than half. Upon ectopic expression of HIF- $1 \alpha, C A 9$ activity markedly increases, and inhibition of MAPKK brings it back down to about one third of the control level. Thus, both HIF-1-dependent and independent components seem to be involved in transmission of regulatory signals by the MAPK pathway to the CA9 promoter.

Interestingly, the MAPK-inhibited cells still retain the capacity to induce $C A 9$ transcription in hypoxia and in high cell density, so additional regulators are apparently involved. In fact, PI3K was already proven to play a role in $C A 9$ control in a dense culture and also seems to participate in HIF-1-mediated induction of $C A 9$ in hypoxia, because its inhibition leads to a decreased level of HIF-1 $\alpha$ protein and consequently to a diminished level of CA IX protein in HeLa cells [8]. Indeed, simultaneous treatment of the dense and hypoxic HeLa cells with the MAPK and PI3K inhibitors had an augmented negative effect on the CA9 promoter activity as well as on CA IX protein expression. The resulting promoter activity and level of the CA IX protein were very low or even absent, clearly indicating that these pathways complement each other in the control of CA9 gene and that they are responsible for a significant part of density-induced as well as hypoxia-induced CA9 expression. 
Previous dissection of the transcriptional factors that execute the hypoxic and density-generated signals by direct binding to the $C A 9$ promoter revealed HIF-1 and SP1 as key players and demonstrated that SP1 activity is required for the full transcription of the $C A 9$ gene under both conditions [18]. Whereas SP1 functioning is obligatory for $C A 9$ induction by density, it seemed to be needed only for an improvement of HIF-1-mediated CA9 transcription under hypoxia [18]. In the present experiments, inhibition of SP1 considerably reduced the hypoxia-induced CA IX protein expression. This effect was stronger after additional inhibition of MAPKK, suggesting that the MAPK pathway, which is constitutively activated in HeLa cells [26], cooperates with SP1 in proper signal transduction to the $C A 9$ promoter. The same explanation possibly applies to SP1's role in MAPK and/or PI3Kmediated $C A 9$ transcription in high cell density. Based on these data, SP1 clearly behaves as an important component of the basal $C A 9$ transcription machinery, which is required for the full performance of both the MAPK and PI3K pathways. This fits well with the current view of SP1 as an acceptor and integrator of signals from these two pathways upon their activation by hypoxia and by extracellular factors $[31,37,38]$.

Taken together, in this work we provided the evidence for an involvement of the MAPK pathway in the regulation of $C A 9$ expression. We demonstrated that this pathway controls the $C A 9$ promoter via both HIF-1-dependent and HIF-1 independent signals and that it works along with the PI3K pathway and SP1 as a downstream mediator of $C A 9$ transcriptional responses to both hypoxia and high cell density. This is an important finding, since activating mutations of various components of both MAPK and PI3K pathways, which occur in many tumor types [39], may upregulate $C A 9$ gene expression inside as well as outside of the hypoxic regions and influence an intratumoral distribution of the CA IX protein. CA IX is functionally implicated in tumor growth and survival $[4,5,40]$, and its increased expression may thus have important consequences for tumor biology.

\section{Acknowledgements}

This work was supported by grants from the Slovak Scientific Grant Agency (VEGA 2/2025, 2/2024), from EU (EUROXY) and from FRSM "La présente publication a été rendue possible grâce à l'Accord qui lie CGRI, le FNRS à l'Académie slovaque des Sciences". R.K. is Research Director of the Belgian FNRS. J.K. was a fellow of InterUniversity Attraction Poles from the Federal Office for Scientific, Technical and Cultural Affairs of Belgium.

\section{References}

[1] C.T. Supuran, Carbonic anhydrases: catalytic mechanisms, distribution and physiological roles, in: C.T. Supuran, A. Scozzafava, J. Conway (Eds.), Carbonic Anhydrase: Its Inhibitors and Activators, CRC Press, Boca Raton, 2004, pp. 1-24.
[2] S. Pastorekova, J. Pastorek, Cancer-related carbonic anhydrase isozymes and their inhibition, in: C.T. Supuran, A. Scozzafava, J. Conway (Eds.), Carbonic Anhydrase: Its Inhibitors and Activators, CRC Press, Boca Raton, 2004, pp. 253-272.

[3] C.P. Potter, A.L. Harris, Diagnostic, prognostic and therapeutic implications of carbonic anhydrases in cancer, Br. J. Cancer 89 (2003) 2-7.

[4] E. Svastova, N. Zilka, M. Zatovicova, A. Gibadulinova, F. Ciampor, J. Pastorek, S. Pastorekova, Carbonic anhydrase IX reduces Ecadherin-mediated adhesion of MDCK cells via interaction with beta-catenin, Exp. Cell Res. 290 (2003) 332-345.

[5] E. Svastova, A. Hulikova, M. Rafajova, M. Zatovicova, A. Gibadulinova, A. Casini, A. Cecchi, A. Scozzafava, C.T. Supuran, J. Pastorek, S. Pastorekova, Hypoxia activates the capacity of tumorassociated carbonic anhydrase IX to acidify extracellular pH, FEBS Lett. 577 (2004) 439-445.

[6] C.C. Wykoff, N.J.P. Beasley, P.H. Watson, K.J. Turner, J. Pastorek, A. Sibtain, G.D. Wilson, H. Turley, K.L. Talks, P.H. Maxwell, C.W. Pugh, P.J. Ratcliffe, A.L. Harris, Hypoxia-inducible expression of tumor-associated carbonic anhydrases, Cancer Res. 60 (2000) $7075-7083$.

[7] J. Lieskovska, R. Opavsky, L. Zacikova, M. Glasova, J. Pastorek, S. Pastorekova, Study of in vitro conditions modulating expression of $\mathrm{MN} / \mathrm{CA}$ IX protein in human cell lines derived from cervical carcinoma, Neoplasma 46 (1999) 17-24.

[8] S. Kaluz, M. Kaluzova, A. Chrastina, P.L. Olive, S. Pastorekova, J. Pastorek, M.I. Lerman, E.J. Stanbridge, Lowered oxygen tension induces expression of the hypoxia marker MN/Carbonic anhydrase IX in the absence of hypoxia-inducible factor $1 \alpha$ stabilization: a role for phosphatidylinositol 3'-kinase, Cancer Res. 62 (2002) 4469-4477.

[9] S. Kaluz, M. Kaluzova, R. Opavsky, S. Pastorekova, A. Gibadulinova, F. Dequiedt, R. Kettmann, J. Pastorek, Transcriptional regulation of the MN/CA9 gene coding for the tumor-associated carbonic anhydrase IX, J. Biol. Chem. 274 (1999) 32588-32595.

[10] M. Kaluzova, S. Pastorekova, E. Svastova, J. Pastorek, E.J. Stanbridge, S. Kaluz, Characterization of the MN/CA9 promoter proximal region: a role for specificity protein (SP) and activator protein 1 (AP1) factors, Biochem. J. 359 (2001) 669-677.

[11] M. Ivan, K. Kondo, H. Yang, W. Kim, J. Valiando, M. Ohh, A. Salic, J.M. Asara, W.S. Lane, W.G. Kaelin, HIF $\alpha$ targeted for VHLmediated destruction by proline hydroxylation: implications for $\mathrm{O}_{2}$ sensing, Science 292 (2001) 464-468.

[12] P. Jaakkola, D.R. Mole, Y.M. Tian, M.I. Wilson, J. Gielbert, S.J. Gaskell, A. von Kriegsheim, H.F. Heberstreist, M. Mukherji, C.J. Schofield, P.H. Maxwell, C.W. Pugh, P.J. Ratcliffe, Targeting of HIF $\alpha$ to the von Hippel Lindau ubiquitilation complex by $\mathrm{O}_{2}$-regulated prolyl hydroxylation, Science 292 (2001) 468-472.

[13] P.C. Mahon, K. Hirota, G.L. Semenza, FIH-1: a novel protein that interacts with HIF-1 $\alpha$ and VHL to mediate repression of HIF-1 transcriptional activity, Genes Dev. 15 (2001) 2675-2686.

[14] G.L. Semenza, Signal transduction to hypoxia-inducible factor 1, Biochem. Pharmacol. 64 (2002) 993-998.

[15] J. Bardos, M. Ashcroft, Hypoxia-inducible factor-1 and oncogenic signaling, BioEssays 26 (2004) 262-269.

[16] E. Laughner, P. Taghavi, K. Chiles, P.C. Mahon, G.L. Semenza, HER2(neu) signaling increases the rate of hypoxia-inducible factor 1alpha (HIF-1alpha) synthesis: novel mechanism for HIF-1-mediated vascular endothelial growth factor expression, Mol. Cell. Biol. 21 (2001) 3995-4004.

[17] D.E. Richard, E. Berra, E. Gothie, D. Roux, J. Pouyssegur, p42/p44 mitogen-activated protein kinases phosphorylate hypoxia-inducible factor $1 \alpha(\mathrm{HIF}-1 \alpha)$ and enhance the transcriptional activity of HIF-1, J. Biol. Chem. 274 (1999) 32631-32637.

[18] S. Kaluz, M. Kaluzova, E.J. Stanbridge, Expression of the hypoxia marker carbonic anhydrase IX is critically dependent on SP1 activity. Identification of a novel type of hypoxia-responsive enhancer, Cancer Res. 63 (2003) 917-922. 
[19] J. Milanini, F. Vinals, J. Pouyssegur, G. Pages, p42/p44 MAP kinase module plays a key role in the transcriptional regulation of the vascular endothelial growth factor gene in fibroblasts, J. Biol. Chem. 273 (1998) 18165-18172.

[20] E. Minet, T. Arnould, G. Michel, I. Roland, D. Mottet, M. Raes, J. Remacle, C. Michiels, ERK activation upon hypoxia: involvement in HIF-1 activation, FEBS Lett. 468 (2000) 53-58.

[21] T. Hofer, I. Desbaillets, G. Hopfl, M. Gassmann, R.H. Wenger, Dissecting hypoxia-dependent and hypoxia-independent steps in the HIF-1 $\alpha$ activation cascade: implications for HIF-1 $\alpha$ gene therapy, FASEB J. 15 (2001) 2715-2717.

[22] S.M. Wood, M.S. Wiesener, K.M. Yeates, N. Okada, C.W. Pugh, P.H. Maxwell, P.J. Ratcliffe, Selection and analysis of a mutant cell line defective in the hypoxia inducible factor $1 \alpha$ subunit (HIF-1 $\alpha$ ), J. Biol. Chem. 273 (1998) 8360-8368.

[23] S. Pastorekova, Z. Zavadova, M. Kostal, O. Babusikova, J. Zavada, A novel quasi-viral agent, MaTu, is a two component system, Virology 187 (1992) 620-626.

[24] J. Zavada, Z. Zavadova, J. Pastorek, Z. Biesova, J. Jezek, J. Velek, Human tumor-associated cell adhesion protein MN/CA IX: identification of M75 epitope and of the region mediating cell adhesion, Br. J. Cancer 82 (2000) 1508-1513.

[25] T.S. Lewis, P.S. Shapiro, N.G. Ahn, Signal transduction through MAP kinase cascades, Adv. Cancer Res. 74 (1998) 49-139.

[26] E. Berra, M.T. Diaz-Meco, J. Moscat, The activation of p38 and apoptosis by inhibition of Erk is antagonized by the phosphoinositide 3-kinase/Akt pathway, J. Biol. Chem. 273 (1998) 10792-10797.

[27] J.J. Haddad, Hypoxia and the regulation of mitogen-activated protein kinases: gene transcription and the assessment of potential pharmacologic therapeutic interventions, Int. Immunopharmacol. 4 (2004) $1249-1285$.

[28] P.J. Kallio, P. Okamoto, S. O’Brien, P. Carrero, Y. Makino, H. Tanaka, L. Poellinger, Signal transduction in hypoxic cells: inducible nuclear translocation and recruitment of the $\mathrm{CBP} / \mathrm{p} 300$ coactivator by the hypoxia-inducible factor-1aplha, EMBO J. 17 (1998) 6573-6586.

[29] N. Sang, D.P. Stiehl, J. Bohensky, I. Leshchinsky, V. Srinivas, J. Caro, MAPK signaling up-regulates the activity of hypoxia-inducible factors by its effects on p300, J. Biol. Chem. 278 (2003) 14013-14019.

[30] E.A. Sheta, H. Trout, J.J. Gildea, M.A. Harding, D. Theodorescu, Cell density mediated pericellular hypoxia leads to induction of HIF-1 $\alpha$ via nitric oxide and Ras/MAP kinase mediated signaling pathways, Oncogene 20 (2001) 7624-7634.

[31] E. Berra, J. Milanini, D.E. Richard, M. Le Gall, F. Vinals, E. Gothie, D. Roux, G. Pages, J. Pouyssegur, Signaling angiogenesis via p42/p44 MAP kinase and hypoxia, Biochem. Phamacol. 60 (2000) 1171-1178.

[32] H. Zhong, K. Chiles, D. Feldser, E. Laughner, E. Hanrahan, M.M. Georgescu, J.W. Simons, G.L. Semenza, Modulation of hypoxiainducible factor $1 \alpha$ expression by the epidermal growth factor/ phosphatidylinositol 3-kinase/PTEN/Akt/FRAP pathway in human prostate cancer cells: implications for tumor angiogenesis and therapeutics, Cancer Res. 60 (2000) 1541-1545.

[33] B.H. Jiang, G. Jiang, J.Z. Zheng, Z. Lu, T. Hunter, P.K. Vogt, Phosphatidylinositol 3-kinase signaling controls levels of hypoxiainducible factor 1, Cell Growth Differ. 12 (2001) 363-369.

[34] D.P. Stiehl, W. Jelkman, R.H. Wenger, T. Hellwig-Burgel, Normoxic induction of the hypoxia-inducible factor $1 \alpha$ by insulin and interleukin-1 $\alpha$ involves the phosphatidylinositol 3-kinase pathway, FEBS Lett. 512 (2002) 157-162.

[35] B.H. Jiang, J.Z. Zheng, M. Aoki, P.K. Vogt, Phosphatidylinositol 3kinase signaling mediates angiogenesis and expression of vascular endothelial growth factor in endothelial cells, Proc. Natl. Acad. Sci. U. S. A. 97 (2000) 1749-1753.

[36] A. Maity, N. Pore, J. Lee, D. Solomon, D.M. O'Rourke, Epidermal growth factor receptor transcriptionally up-regulates vascular endothelial growth factor expression in human glioblastoma cells via a pathway involving phosphatidylinositol 3-kinase and distinct from that in hypoxia, Cancer Res. 60 (2000) 5879-5886.

[37] Z.Y. Jiang, Z. He, B.L. King, T. Kuroki, D.M. Opland, K. Suzuma, I. Suzuma, K. Ueki, R.N. Kulkarni, C.R. Kahn, G.L. King, Characterization of multiple signaling pathways of insulin in the regulation of vascular endothelial growth factor expression in vascular cells and angiogenesis, J. Biol. Chem. 278 (2003) 31964-31967.

[38] G.W. Huang, L.Y. Yang, W.Q. Lu, Effects of PI3K and p42/p44 MAPK on overexpression of vascular endothelial growth factor in hepatocellular carcinoma, World J. Gastroenterol. 10 (2004) 809-812.

[39] B. Vogelstein, K.W. Kinzler, Cancer genes and the pathways they control, Nat. Med. 10 (2004) 789-799.

[40] N. Robertson, C. Potter, A.L. Harris, Role of carbonic anhydrase IX in human tumor cell growth, survival and invasion, Cancer Res. 64 (2004) 6160-6165. 